\title{
GRAZER \\ PHILOSOPHISCHE STUDIEN
}

INTERNATIONALE ZEITSCHRIFT FÜR

ANALYTISCHE PHILOSOPHIE

herausgegeben von

RUDOLF HALLER

VOL 47 - 1994 


\section{GRAZER PHILOSOPHISCHE STUDIEN}

\section{Herausgeber/Editor: Rudolf HALLER}

Board of Consulting Editors: H. ALBERT (Mannheim, BRD), D. FØLLESDAL (Oslo, Norway), P. GOCHET (Liège, Belgium), R. HILPINEN (Turku, Finland), J. HINTIKKA (Boston, Massachusetts, USA), R. KAMITZ (Graz: Austria), J. KIM (Ann Arbor, Michigan, USA), S. KÖRNER (Bristol, Greal Britain), F. v. KUTSCHERA (Regensburg, BRD), K. LAMBERT (Irvine, California, USA), K. LEHRER (Tucson, Arizona, USA), J.L. MACKIE † (Oxford, Great Britain), B. McGUINNESS (Siena, Italy), J. MORAVCSIK (Stanford, USA), E. MORSCHER (Salzburg, Austria), G. PATZIG (Göttingen, BRD), M. PRZEŁECKI (Warsawa, Poland), W. RÖD (Innsbruck Austria), W. STEGMÜLLER † (München, BRD), J. VUILLEMIN (Paris France), P. WEINGARTNER (Salzburg, Austria)

\section{Managing Editor: Ulf HÖFER}

Die GRAZER PHILOSOPHISCHE STUDIEN erscheinen zweimal jahrlich in Bänden zu je wenigstens 200 Seiten Umfang. Bestellungen sind an den Buchhandel oder direkt an den Verlag (Editions Rodopi B.V., Keizersgracht 302-304, 1016 EX Amsterdam) zu richten.

MANUSKRIPTE in deutscher, englischer oder französischer Sprache, in zweifacher Ausfertigung, wenn möglich zusammen mit DISKETTEN im MSDOS oder McIntosh Format, sonstige REDAKTIONELLE KORRESPONDENZ und REDAKTIONSEXEMPLARE sind zu senden an "Grazer Philosophische Studien", Institut für Philosophie, Universität Graz, A-8010 Graz, Austria.

Der Verlag stellt jedem Autor 25 Separata unentgeltlich zur Verfügung. Seitens des Herausgebers wird jeder eingereichte Aufsatz einem Mitglied aus dem Board of Consulting Editors zur Begutachtung vorgelegt; der Herausgeber entscheidet aufgrund dieses Gutachtens über die Annahme des Artikels. Aufsätze wie Rezensionen können nur in druckfertigem Zustand und in zweifacher Ausfertigung übernommen werden. Aufsätze dürfen den Umfang von höchstens 30 Maschinschreibseiten zu 30 Zeilen nicht überschreiten. Rezensionen werden nur nach Einladung angenommen. Handschriftliche Eintragungen und Änderungen sind in allen Typoskripten $\mathrm{zu}$ vermeiden. Nachträgliche Korrekturen können nicht berücksichtigt werden. Die Anmerkungen sollen in fortlaufender Nummerierung getrennt dem Text beigeschlossen werden. Bei Aufsätzen ist eine 6-8-zeilige Zusammenfassung beizufügen. Im allgemeinen gelten für den Herausgeber wie für die Verfasser von Beiträgen die "Guidelines for the Handling of Manuscripts" nach dem Journal of Philosophy 72(1975), 466-7. 


\title{
PARMENIDES UND DIE LOGIK DER EXISTENZ
}

\author{
Uwe MEIXNER \\ Universität Regensburg
}

I. Der Schwierigkeit und dem fragmentarischen Zustand des gut 2500 Jahre alten Textes von Parmenides' Lehrgedicht zum Trotz läßt sich doch sagen, daß Kernaussagen dieses Gedichtes die folgenden sind:

(1) Sein (Seiendes) ist. (Frag. 6,1)

(2) Nichtsein (Nichtseiendes) ist nicht. (Frag. 7,1; Frag. 8,46)

(3) Nichts ist nicht. (Frag. 6,2)

(4) Das Nichtseiende kann weder erkannt noch ausgesprochen werden. (Frag. 2,7/8)

(5) Nichts anderes ist außer dem Seienden. (Frag. 8,36/37)

(6) Darum wird alles Name sein, was die Sterblichen gesetzt haben, überzeugt, es sei wahr; Werden und Vergehen, Sein und Nichtsein, und Verändern des Ortes und Wechseln der leuchtenden Farbe. (Frag. 8,38-41)

(7) Entstand Seiendes, so ist es nicht, auch nicht, wenn es einmal sein wird. (Frag. 8,20)

(8) Seiendes muß entweder ganz und gar sein oder überhaupt nicht. (Frag. 8,11)

(9) Seiendes ist Eines. (Frag. 8,6)

(10) Nicht soll dich die vielerfahrene Gewohnheit auf diesen Weg drängen, anzuwenden das blicklose Auge, das dröhnende $\mathrm{Ge}-$ hör. (Frag. 7,3/4)

Das Folgende stellt eine Interpretation dieser Aussagen im Lichte der modernen Logik und formalen Ontologie dar. Überraschenderweise wird sich dabei zeigen, daß Parmenides auch dem analytisch geschulten Ontologen der Gegenwart neue Perspektiven zu eröffnen vermag. Auf diesen wenigen Seiten kann es aber nicht darum gehen, 
eine Gesamtinterpretation Parmenides' zu liefern. Auslassungen sind daher notwendig. Zur Diskussion steht allein der Kern seines Lehrgedichts, der philosophiegeschichtlich insofern am bedeutsamsten war, als er die meisten Denker dazu gedrängt hat, Parmenides $\mathrm{zu}$ antworten.

II. Es gibt eine Deutung, in der (1) und (2) trivialerweise wahr (und logisch äquivalent) sind:

D1(1) Seiendes ist seiend, d.h. alles Seiende ist seiend. Formal: $\forall \mathrm{x}(\mathrm{S}(\mathrm{x}) \supset \mathrm{S}(\mathrm{x}))$

D1(2) Nichtseiendes ist nicht seiend, d.h. alles Nichtseiende ist nicht seiend.

Formal: $\forall \mathrm{x}(\neg \mathrm{S}(\mathrm{x}) \supset \neg \mathrm{S}(\mathrm{x}))$

Bei D1(1) und D1(2) handelt es sich um elementare Tautologien.

Die erste Hauptinterpretationshypothese, von der ich ausgehe, lautet: Mit (5) meint Parmenides dasselbe wie mit (1) und (2) zusammen. Man kann folgerichtig (5) auch trivial tautologisch lesen:

D1(5) Nichts anderes ist seiend außer dem Seienden, d.h. Seiendes ist seiend, und nichts Nichtseiendes ist seiend.

Formal: $\forall \mathrm{x}(\mathrm{S}(\mathrm{x}) \supset \mathrm{S}(\mathrm{x})) \wedge \neg \exists \mathrm{x}(\neg \mathrm{S}(\mathrm{x}) \wedge \mathrm{S}(\mathrm{x}))$, logisch äquivalent: $\forall x(S(x) \supset S(x)) \wedge \forall x(\neg S(x) \supset \neg S(x))$

Meine zweite Hauptinterpretationshypothese lautet: Mit (3) meint Parmenides dasselbe wie mit (2). Aber bei (3) bringt derselbe Interpretationsschritt - die Ergänzung von ,,ist“ $\mathrm{zu}$,,ist seiend“, bzw. von ,,ist nicht" zu , ist nicht seiend" - eine nichttriviale Aussage hervor:

D2(3) Nichts ist nicht seiend, d.h. alles ist seiend.

Formal: $\neg \exists \mathrm{x} \neg \mathrm{S}(\mathrm{x})$, logisch äquivalent: $\forall \mathrm{xS}(\mathrm{x})$

Mit der trivialen Deutung von (2), D1(2), kann man (3) nun nur 
harmonisieren, wenn man über den gegebenen Interpretationsschritt hinaus die Lesart von ,,nichts“ $(\mu \eta \delta \varepsilon v)$ als Quantor aufgibt und es stattdessen als anderes Wort für, ,Nichtseiendes“ ( $\mu \eta \varepsilon 0 v)$ auffaßt, d.h. von der Deutung D2(3) zu der Deutung D1(3) übergeht - eine Vorgehensweise, die sich, wenn keine zusätzlichen Informationen vorlägen, durchaus noch rechtfertigen ließe.

Nun sehen wir aber, daß Parmenides aus (5) eine nichttriviale Folgerung, nämlich (6): die Leugnung jeder Art von Veränderung zieht ((6) folgt textlich zwar nicht unmittelbar auf (5); daß ein Schluß von (5) auf (6) intendiert ist, ist aber unverkennbar). Aus Trivialem folgt jedoch nur Triviales. Demnach kann die Deutung D1(5), die (5) trivial werden läßt, nicht richtig sein - es sei denn man schriebe Parmenides einen groben (allzu groben!) logischen Schnitzer zu:

Als Alternative zu D1(3) kommt D2(3) in Frage. Wenn wir D2(3) als Ausgangspunkt nehmen, erhalten wir dann schließlich eine adäquatere Deutung von (5)? Da (3) und (2) dasselbe besagen, muß dann (2) ebenso gedeutet werden, wie (3) durch D2(3) gedeutet wird, nämlich als logisch äquivalent mit ,Alles ist seiend“", d.h. als $\neg \exists \mathrm{x} \neg S(\mathrm{x})$; (1) und (5) lassen sich mit dieser Deutung harmonisieren: Man ersetze das ,,ist“ in (1), (2) und (5) durch ,,es gibt“ und lese diesen Ausdruck nicht etwa im Sinne von ,,ist seiend", sondern im Sinne des Existenzquantors $\exists$ :

D2(1) Seiendes gibt es, d.h. es gibt etwas, das seiend ist.

Formal: $\exists \mathrm{xS}(\mathrm{x})$

D2(2) Nichtseiendes gibt es nicht, d.h. nichts ist nicht seiend.

Formal: $\neg \exists \mathrm{x} \neg \mathrm{S}(\mathrm{x})$, logisch äquivalent: $\forall \mathrm{xS}(\mathrm{x})$

D2(5) Nichts anderes gibt es außer dem Seienden, d.h. Seiendes gibt es, und Nichtseiendes gibt es nicht.

Formal: $\exists \mathrm{xS}(\mathrm{x}) \wedge \neg \exists \mathrm{x} \neg \mathrm{S}(\mathrm{x})$

(D2(5) ist wohl auch eine mögliche Deutung von Frag. 2,3.)

Wenn es nun auch keineswegs als evident gelten kann, wie aus (5) in der Deutung D2(5) (6) folgen kann, so ist dies doch jedenfalls etwas, das der näheren Untersuchung bedarf; hingegen ist (6) aus 
(5) in der Deutung D1(5) evidentermaßen nicht folgerbar.

D1(5) kann Parmenides mit (5) nicht gemeint haben, oder vorsichtiger: nicht univok gemeint haben. Wenn es ihm nämlich um die evidente Richtigkeit von (5) (und damit von (1), (2) und (3)) ging, so könnte ihm durchaus die Deutung D1(5) präsent gewesen sein; wenn es ihm aber darum ging, (6) aus (5) zu folgern, so muß ihm im Gegenteil die (übrigens noch weiter zu spezifizierende) Deutung D2(5) vorgeschwebt haben. Daß er dann also zwischen zwei Deutungen schwankte, entging ihm, da er nicht über die begrifflichen Mittel verfügte, sich den Unterschied zwischen beiden klar zu machen.

Es gibt aber noch eine dritte Deutung D3. Man erhält sie, wenn man in (1), (2), (3) und (5) ,,ist" durch ,,existiert" ersetzt und dieses Wort nicht im Sinne des Existenzquantors (D2) oder der trivialen Subsumtion (D1) nimmt, sondern im Sinne des auf Eigennamen angewendeten Existenzprädikats (wie z.B. in ,Hans existiert“). Es ist dann in (1), (2) und (3) von zwei Objekten die Rede: dem Sein (dem Seienden) und dem Nichtsein (dem Nichtseienden, dem Nichts), von denen ausgesagt wird, da $B$ das eine existiert, das andere aber nicht. Schwerlich läßt sich jedoch (5) in dieser Weise lesen, das nach der ersten Hauptinterpretationshypothese nichts weiter als die Konjunktion von (1) und (2) sein müßte; denn das ,,nichts“

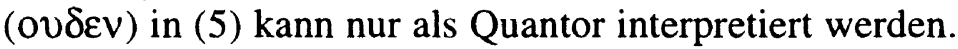

D3 paßt zu (9) (im Sinne von ,,Das Seiende ist ein unteilbares Objekt [Individuum]") und zu dem, was Parmenides außer den angeführten Kernaussagen in seinem Lehrgedicht sonst über das Seiende sagt; er spricht von ihm als von einem einzelnen (quasigöttlichen) Objekt. Jedoch läßt sich auch D2 mit (9) (dann im Sinne von „Es gibt genau ein Seiendes [nicht viele Seiende]“) und den sonstigen Aussagen harmonisieren (denn wenn es genau ein Seiendes gibt, dann kann man von dem Seienden als einem einzelnen Objekt sprechen).

Gegen die Deutung D3 spricht die Kernaussage (4): Nach D3 ist im Lehrgedicht von dem Nichtseienden (als einzelnem Objekt) die Rede, d.h. auf es wird der Intention nach benennend Bezug genommen. Dies verbietet aber gerade (4), wenn es sagt, daß das Nichtseiende nicht ausgesprochen werden kann; d.h. auf das Nichtseiende kann nach (4) - jedenfalls gemäß einer sehr plausiblen Inter- 
pretation - nicht benennend Bezug genommen werden. (Kahn bietet für Frag. 2,8 in ,,The Thesis of Parmenides“, S. 713: ,, nor can you point it out", entsprechend auch Gallop und Austin; Heitsch übersetzt ,,[kannst du weder erkennen] noch aufzeigen" in Parmenides: Die Fragmente, S.15; diese von der traditionellen Übersetzung abweichenden Wiedergaben stützen unsere Interpretation.) Und das darf dann konsistenterweise natürlich auch in (4) selbst nicht intendiert sein; d.h. ,,das Nichtseiende " fungiert in (4) als bezugsloser (aber sinnvoller) Name, oder aber bedeutet soviel wie ,, alles Nichtseiende" (wird also prädikativ: als Prädikat im logischen Sinn verwendet).

Im Sophistes, 238c-239a, geht der eleatische Fremde offenbar von der Deutung D3 aus und behauptet die Widersprüchlichkeit der parmenideischen Thesen (und er hat dabei insbesondere auch die Selbstwidersprüchlichkeit von (4) im Sinn!), solange man mit dem Namen ,,das Nichtseiende“ das sinngemäß intendierte Objekt benennen will; da die Konzeption leerer Namen ihm fremd ist, bleibt zur Vermeidung eines Widerspruchs nur, den Gebrauch des angeführten Namens und jedes anderen für diesen substitutierbaren Namens zu vermeiden: (239a), ,Fremder: Und wir behaupten doch, wer richtig reden solle, dürfe es weder als eins noch als vieles bestimmen, noch es überhaupt auch nur ,es' nennen; denn schon durch diese Angabe würde er es als Eins angeben."

Die Sophistes-Stelle zeigt, daß die Deutung D3 keine neuartige ist, sondern bereits in der Antike tatsächlich erwogen wurde (zumindest von Platon selbst, wahrscheinlich aber nicht nur von ihm). Ist sie aber deshalb diejenige, die Parmenides selbst intendierte? Wie gesagt, bei ihr verwickelt sich Parmenides - wie Platon scharfsinnig sah - in einen Widerspruch, was man ihm ohne Not nicht unterstellen sollte, und Platon selbst gibt indirekt $\mathrm{zu}, \mathrm{da} ß$ auch er nur über Interpretationshypothesen bzgl. dessen verfügt, was Parmenides meinte; siehe Sophistes, 242b-243b.

Nun läßt sich D3 verfeinern, indem man sagt, was von ,,das Nichtseiende" in (4) gilt, gelte von den Vorkommnissen der Ausdrücke ,,Nichtsein“, ,,Nichtseiendes" im Lehrgedicht überhaupt: sie sind sämtlich der Intention nach leere Namen, und wenn Parmenides sagt, daß das Nichtsein nicht existiert, so meint er schlicht, daß der Name ,,das Nichtsein“ nichts bezeichnet. Ganz abgesehen da- 
von, wie weit diese Interpretationshypothese ansonsten trägt, die Konzeption leerer Namen in dennoch wahren Sätzen ist von außerordentlicher semantischer Sophistikation, und man wird sie Parmenides nicht zuschreiben können. Sehr viel plausibler ist demgegenüber doch die prädikative, eben nicht nominale (d.h. sie nicht zu Eigennamen machende) Auffassung von „Sein" und „Nichtsein“" (wie sie den Deutungen D1 und D2 zugrundeliegt).

Übrigens kann man auch, anders als in D1 geschehen, ,, ist seiend" (für $\varepsilon \sigma \tau \imath$ ), bzw. ,,ist nicht seiend" (für ovK $\varepsilon \sigma \tau \imath$ ) als auf Eigennamen angewendetes Prädikat auffassen; das ausgesagte Prädikat kommt dann als kennzeichnendes Prädikat (als Prädikat, mittels dessen die - eindeutig gemeinte - Kennzeichnung geschieht) schon in den Eigennamen (,,das Seiende“ bzw. ,,das Nichtseiende“) vor. Dies gibt (1) und (2) abermals einen tautologischen Klang; man kann aber nicht sagen, daß sie auch Tautologien sind: Nach der Russellschen Standardtheorie der Kennzeichnung sind beide Sätze in der nun betrachteten Deutung falsch, wenn es weder genau ein Seiendes noch genau ein Nichtseiendes gibt. Und daß es genau ein Seiendes und genau ein Nichtseiendes gibt, was beide Sätze (in der betrachteten Deutung) wahı machen würde, kann man gewiß nicht als trivialerweise logisch wahr ansehen. - Es ist freilich offensichtlich, daß sich gegen die zuletzt betrachtete Deutung dieselben Einwände wie gegen D3 vorbringen lassen.

III. Gemäß D2 ist (5) (und damit (1), (2), (3)) zwar nicht trivialerweise wahr, wohl aber kann man das Prädikat ,, $\mathrm{x}$ ist seiend" so deuten, daß die Aussage $\exists \mathrm{xS}(\mathrm{x}) \wedge \neg \exists \mathrm{x} \neg \mathrm{S}(\mathrm{x})[\forall \mathrm{xS}(\mathrm{x})]$ eine Wahrheit der klassischen Logik wird:

\section{Def1 $S(x):=\exists y(x=y)$}

Laut Definition 1 ist $x$ genau dann seiend, wenn ein Objekt (im weitesten Sinne: eine - nichtsprachliche - Entität) mit ihm identisch ist. Nun sind es Gesetze der klassischen Logik (mit Identität), daß ein Objekt mit einem Objekt identisch ist, und daß jedes Objekt mit einem Objekt (nämlich sich selbst) identisch ist: $\exists x \exists y(x=y) \wedge$ $\forall \mathrm{x} \exists \mathrm{y}(\mathrm{x}=\mathrm{y})$. Also ergibt sich mit Def $1 \exists \mathrm{xS}(\mathrm{x}) \wedge \forall \mathrm{xS}(\mathrm{x})$. Falls also 
Parmenides ,,seiend“ im Sinne von Def1 verstanden hat, so gehört zu seinen logischen Entdeckungen nicht nur das Tertium-non-Datur $(A \vee \neg A$; explizit formuliert in Frag. 8,16), der Satz vom Widerspruch $(\neg(A \wedge \neg A)$; siehe die Polemik in Frag. 6,8/9) und der Beweis

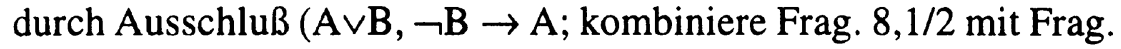
$2,3 / 5 / 6)$, sondern eben auch das angeführte identitätslogische $\mathrm{Ge}$ setz.

In der sogenannten Freien Logik, in der die Interpretation der logischen Konstanten nicht erfordert, daß der Grundbereich der Sprache nichtleer sei, ist $\exists x S(x)$ (im Sinne von Def1) übrigens nicht mehr logisch wahr (wohl aber bleibt dies $\neg \exists x \neg S(x)$ !); der Grundbereich könnte nämlich leer sein, und dann wäre $\exists x S(x)$ falsch. Das ändert nichts daran, da $\exists \mathrm{xS}(\mathrm{x})$ (im Sinne von Def1) immer noch faktisch wahr ist. Wie könnte man daran zweifeln?

Hat nun aber Parmenides ,,seiend“" im Sinne von Def1 verstanden? Jedenfalls gibt es starke Anzeichen dafür, daß gemäß seiner Deutung , $\mathrm{x}$ ist seiend" notwendigerweise denselben Umfang hat wie nach Def1. Gemäß Def1 wird ja ,,seiend“ in einem weitest möglichen Sinn definiert, und auch Parmenides impliziert eine sehr weite Bestimmung des Seienden. Nach (4) kann Nichtseiendes (alles Nichtseiende) weder erkannt noch benannt werden; folglich ist alles, was erkannt oder benannt werden kann, seiend. Hier haben wir also eine sehr schwache hinreichende Bedingung für seiend; zumal dann, wenn man ,kann“ im schwächst möglichen Sinn nimmt, wie es angesichts des emphatischen Charakters von Frag. 2,7/8, wo Erkenn- und Benennbarkeit offenbar in jedem noch so weiten Sinn ausgeschlossen werden soll, sicherlich erlaubt ist. Tut man nun dies, so entfallen auch alle Bedenken dagegen, die hinreichende Bedingung zudem als notwendige zu betrachten. Auch Parmenides, so kann man sagen, deutet demnach (an manchen Stellen in seinem Lehrgedicht!) ,,seiend" in einem sehr weiten Sinn (bringt er dies nicht - unzulänglich - zum Ausdruck mit seiner folgenschweren Behauptung in Frag. 3, daß Denken und Sein dasselbe ist?), und man braucht nicht zu zögern, diesen mit dem dem Prädikat , $\mathrm{X}$ ist seiend“ in Def1 verliehenen Sinn extensional zu identifizieren; d.h. der Begriff, im weitesten Sinne erkennbar oder benennbar $\mathrm{zu}$ sein, trifft notwendig auf dieselben Objekte zu wie der Begriff, mit einem Objekt identisch zu sein. (Extensional identische, d.h. 
extensional ununterscheidbare Begriffe, sind, wie auch im vorliegenden Fall, noch nicht identisch simpliciter; anderes Beispiel: der Begriff, ein gleichseitiges Dreieck zu sein, und der Begriff, ein gleichwinkeliges Dreieck zu sein.)

IV. Andererseits ist aber nicht zu sehen, wie (6) daraus folgen soll, daß etwas (im weitesten Sinne) erkennbar oder benennbar ist und nichts weder erkennbar noch benennbar ist; d.h. wie sich (6) aus D2(5) in der durch (4) nahegelegten eben betrachteten spezifischeren Deutung ergeben könnte.

Es kann kaum anders sein: Parmenides geht beim Übergang von (5) $\mathrm{zu}$ (6) auch von einem sehr weiten (d.h. inhaltsarmen oder schwachen) Seinsbegriff zu einem sehr viel engeren (d.h. inhaltsreicheren oder stärkeren) über - ohne sich natürlich dessen bewußt zu werden. Er unterschied noch nicht engere und weitere Seinsbegriffe, und ebensowenig absolute und zeitlich oder gradmäßig relativierte. Wie (7) und (8) deutlich machen, hätte er sich auch gegen jeden Versuch einer solchen Differenzierung zur Wehr gesetzt. In dieser Haltung ist Parmenides, wie es scheint, keineswegs längst überholt. Der moderne Ontologe E. Tegtmeier schreibt wie ein wiedererstandener Parmenides in seinem 1992 erschienen Buch Grundzüge einer kategorialen Ontologie:

Denn es dürfte schwer sein, mit einer Abstufung des Seins, der Existenz einen klaren Sinn zu verbinden. (S. 23)

Unabhängig von der Ontologie, die ich vertrete, halte ich die Rede von zeitlicher Existenz, ebenso wie die von verschiedenen Weisen oder Graden der Existenz, einfach für unsinnig. Entweder etwas existiert oder es existiert nicht. Dazwischen findet man nichts, weder geringere Grade der Existenz, noch ein Nichtmehr-existieren. (S.145f)

Welcher ist aber nun der Seinsbegriff, zu dem Parmenides unversehens hinübergleitet? Ich werde zeigen, daß es der des Aktualseins (Realseins, Wirklichseins) sein muß - einen Begriff, den explizit zuerst Aristoteles in die Philosophie einführte, und zwar u.a. um eben die Aporie zu beseitigen, die Parmenides mit seiner Argumentation gegen das Vorhandensein jeglicher Veränderung erzeugt hatte. 
Während D2(1) und D2(2) als wahr gelten müssen, wenn man ,,seiend“ in einem sehr weiten Sinn nimmt - wie das ,,örtlich“" auch Parmenides tut -, sieht die Sache anders aus, wenn man definiert:

\section{Def2 $\quad \mathrm{S}(\mathrm{x}):=\mathrm{x}$ ist aktual (real)}

Zwar ist $\exists \mathrm{xS}(\mathrm{x})$ nach wie vor gesichert (zumindest daran, daß man selbst aktual ist, ist nicht zu zweifeln), aber $\neg \exists x \neg S(x)$ wird nun zweifelhaft. Ist tatsächlich alles aktual? Oder ist doch einiges nichtaktual, d.h. potentiell? Kraft seiner Unterscheidung verschiedener Seinsbegriff sah Aristoteles in direkter Antwort auf Frag. 8,6-10 und 8,12/13 im Nichtaktualen, aber darum doch nicht gänzlich Nichtseienden gerade das Etwas, das die ontologisch notwendige Grundlage des Werdens bildet. (Vergl. hierzu W. K. Guthrie, $A$ History of Greek Philosophy, Bd. 6, S. 120f.) Wie wir sehen werden, hat er damit recht.

Man sieht, daß man sich der Prämisse von Parmenides' Argumentation - (1) und (2), d.h. (5) - mittels Def1 oder der damit notwendig äquivalenten Seinsbestimmung, die sich dem Text entnehmen läßt, versichern kann; diese Definition ermöglicht es aber nicht, aus der Prämisse die Folgerung (6) zu ziehen. Dagegen erlaubt es Def2 tatsächlich, aus (5) (6) zu folgern - wie wir gleich sehen werden -, aber (5) wird bei ihr problematisch. In jedem Fall vermag Parmenides' Argumentation nicht zu überzeugen: im einen Fall (bei Def1), weil sie unschlüssig, im anderen Fall (bei Def2), weil ihr Fundament zweifelhaft ist.

Nicht wenige moderne Ontologen sähen dies aber anders. Der Aktualismus, der Standpunkt, daß es nur Reales gibt, ist immer noch weit verbreitet. Ein Aktualist würde also Parmenides' Prämisse (5) in der durch Def2 spezifizierten Deutung D2 akzeptieren, aber leugnen, daß daraus (6) folgt. Dies ist ein Irrtum. Aus der Behauptung, daß es nur Reales gibt, folgt, daß es keine Veränderung jeglicher Art gibt; es folgt außerdem aus ihr (9) in der entsprechenden Deutung (nämlich als ,Es gibt genau ein Seiendes, d.h. Aktuales"). Diese Tatsache ist der Grund dafür, Parmenides zuzuschreiben, daß er von einem Seinsbegriff à la Def1 zu dem gemäß Def2 übergeht. Die Folgerbarkeit hat Parmenides gewiß nicht clare et distincte, wohl aber ungefähr eingesehen; man vergleiche dazu 
Frag. 8,613 mit den in V. angegebenen Ableitungen.

Sie zeigt aber gleichzeitig - und das ist ein Resultat, das nicht nur von philosophiehistorischem Interesse ist -, daß der Aktualismus nicht bloß zweifelhaft, sondern falsch ist. Denn man wird doch, mit der Folgerbarkeit konfrontiert, nicht wie Parmenides bereit sein, das Zeugnis der Sinne für das Gegebensein von Veränderung und Vielheit als vollständig illusorisch hinzustellen (siehe (10)), was eine totale Entwertung der Erfahrung als Erkenntnisquelle bedeuten muß.

V. Wie folgt aber nun aus ,Es gibt nur Aktuales (Reales)“ (formal: $\exists x \operatorname{Act}(x) \wedge \forall x \operatorname{Act}(x))$, daß es keine Veränderung gibt, bzw. daß genau ein Objekt aktual ist (formal: $\exists x \operatorname{Act}(\mathrm{x}) \wedge \forall \mathrm{x} \forall \mathrm{y}(\operatorname{Act}(\mathrm{x})$ $\wedge \operatorname{Act}(\mathrm{y}) \supset \mathrm{x}=\mathrm{y}))$ ?

(a) Angenommen das Objekt $\mathrm{x}$ ist bzgl. der Zeitpunkte $\mathrm{t}$ und $\mathrm{t}^{\prime}$ in der Hinsicht $f$ verändert; das bedeutet, daß $x$ zu t $f$ ist, $\mathrm{zu}^{\prime} \mathrm{t}^{\prime}$ aber nicht; oder daß x zu t nicht $f$ ist, zu $t^{\prime}$ aber schon. In beiden Fällen besteht also ein gewisser Sachverhalt nicht: daß $x$ zu t' $\mathrm{f}$ ist, bzw. $\mathrm{da} \times$ × zu t $\mathrm{f}$ ist; also gibt es einen nichtbestehenden Sachverhalt; also gibt es Nichtreales. Es ist jedoch laut Annahme alles real. Folglich gibt es eben kein $x$, keine Zeitpunkte $t$ und $t^{\prime}$ und keine Hinsicht $f$, so daß gelten würde: $x$ ist bzgl. $t$ und $t^{\prime}$ in $f$ verändert. Also gibt es keine Veränderung.

(b) Angenommen $x$ und y sind zwei verschiedene Objekte. Dann muß es eine Hinsicht $f$ geben, in der sie sich unterscheiden; d.h. es muß gelten $x$ ist $f$, und $y$ nicht, oder $y$ ist $f$, und $x$ nicht. In beiden Fällen besteht also ein gewisser Sachverhalt nicht: daß y f ist, bzw. $\mathrm{da}$ x $\mathrm{f}$ ist; also gibt es einen nichtbestehenden Sachverhalt; also gibt es Nichtreales. Laut Annahme ist aber alles real. Folglich gibt es eben keine zwei verschiedenen Objekte (d.h. $\forall \mathrm{x} \forall \mathrm{y}(\mathrm{x}=\mathrm{y})$ ); da es aber ein reales Objekt $(\exists x \operatorname{Act}(\mathrm{x}))$ gibt, folgt hiermit, daß es genau ein reales Objekt gibt.

Wie alle Argumentationen haben auch diese beiden Deduktionen ihre Voraussetzungen: Es gelten die Gesetze der klassischen Logik, insbesondere das der Existenz(quantor)generalisierung: Der Schluß von $A$ [a] auf $\exists x A[x]$ ist stets gültig; $A[a]$ ist hierbei ein Satz, der an gewissen Stellen den Term a enthält. (Auch freie Variablen sind 
Terme, Terme enthalten freie Variablen, oder auch nicht, sie sind einfach, oder auch komplex: Jeder benennende Ausdruck ist ein Term.) Einfachste Daß-Sätze der Gestalt ,,daß a (zu $\tau) \mathrm{F}$ ist" werden als komplexe Terme für Sachverhalte aufgefaßt (deren Bezug - jeweils ein Sachverhalt - im Grundbereich der Objekte im weitesten Sinne liegt, über den quantifiziert wird). (Warum nur einfachste? Dagegen läßt sich vom Standpunkt der ontologischen Sparsamkeit am wenigsten sagen und mehr wird hier auch nicht benötigt. Außerdem vermeiden wir damit, daß sich, daß das Seiende ist und $d a \beta$ das Nichtseiende nicht ist, als zwei bestehende, d.h. aktuale Sachverhalte erweisen, wo wir doch gerade aufgrund ihrer bewiesen haben, daß es nur eine aktuale Entität gibt.) Somit ist der $\mathrm{Satz}$,,Daß a $(z u \tau)$ F ist, ist ein Sachverhalt" stets wahr, und es gilt stets der Schluß von , $\mathrm{Daß}$ a $(\mathrm{zu} \tau) \mathrm{F}$ ist, ist ein nichtbestehender Sachverhalt “ auf ,Es gibt einen nichtbestehenden Sachverhalt". Auch wenn man zur Freien Logik übergeht, in der das Gesetz der Existenzgeneralisierung nicht uneingeschränkt gilt, wird man doch an der generellen Gültigkeit der Existenzgeneralisierung bzgl. einfachster komplexer Sachverhaltsterme (einfachster Daß-Sätze) festhalten müssen; es sei denn man könnte zeigen, daß auch die einfachsten Daß-Sätze gelegentlich nicht referieren. - Außerdem gelten:

$(\alpha) \mathrm{Da} \beta$ a $(z u \tau) F$ ist, ist ein bestehender Sachverhalt (Tatsache) genau dann, wenn a (zu $\tau) \mathrm{F}$ ist;

$(\beta) \mathrm{p}$ ist ein bestehender Sachverhalt genau dann, wenn $\mathrm{p}$ ein aktualer (realer) Sachverhalt ist.

Für die zweite Deduktion ist zudem speziell vorausgesetzt, daß sich verschiedene Objekte in gewisser Hinsicht unterscheiden müssen. (Der erste Schluß in der ersten Deduktion ist vollkommen unproblematisch, und das ihn ermöglichende Gesetz braucht daher nicht unter die Voraussetzungen gezählt zu werden.)

Diese logischen Voraussetzungen sind nicht unantastbar, besitzen aber eine große Evidenz, die sich nicht so leicht aushebeln läßt. Insbesondere waren sie in ihrer Einfachheit schon den (alten) Griechen vertraut (was nicht bedeutet, daß sie sie hätten explizit formulieren können). Sie konnten mittels $\varepsilon \sigma \tau \imath$ ohne prädikative Ergänzung u.a. auch ausdrücken, daß etwas eine Tatsache, d.h. ein beste- 
hender Sachverhalt ist (dazu ausführlicher in VII.). Das bedeutet, daß Parmenides das Prinzip $(\alpha)$ kaum gedanklich fern gelegen haben dürfte; das Prinzip ( $\beta$ ) aber beschreibt, wenn man ,,aktual“" durch ,,seiend“ (im Sinne von , ,aktual“" verstanden) ersetzt, den beschriebenen Gebrauch von $\varepsilon \sigma \tau \imath$ so nahe, wie das im Deutschen überhaupt möglich ist. Der schwächste Punkt ist noch, daß alle Terme der Gestalt ,,daß a (zu $\tau) \mathrm{F}$ ist" auf Sachverhalte referieren, oder mit anderen Worten, daß der Satz , $\mathrm{Da} \beta \mathrm{a}(\mathrm{zu} \tau) \mathrm{F}$ ist, ist ein (bestehender oder auch nicht bestehender) Sachverhalt" stets wahr ist. Aber auch davon gingen die Griechen ohne weiteres aus im Sinne des Prinzips der naiven Semantik: Wo ein Name, da auch ein benanntes Objekt (ein Etwas, ein Eins). Wie hätte es Platon sonst zum Problem werden können, daß es offenbar Falsches (benannte nichtbestehende Sachverhalte) gibt, während doch, was falsch ist, nichtseiend ist, und es Nichtseiendes nicht gibt, wie Parmenides so schwer widerlegbar behauptet hatte. (Vergl. hierzu Sophistes, 236e-237b, 241d,e.)

VI. Merkwürdig ist, daß Parmenides sich über manche naheliegenden Schwierigkeiten für seine Position offenbar keine Gedanken gemacht hat (soweit wir dies beurteilen können). Wenn Seiendes Eines ist, was ist dann mit ihm selbst, Parmenides? Ist er selbst das Seiende? Oder ist er etwa nicht seiend? Wäre dies letztere der Fall, so gäbe es etwas Nichtseiendes; solches aber gibt es doch nicht. Es bleibt ihm nichts anderes übrig, als mit dem Seienden identisch zu sein. Die Absurdität dieser solipsistischen Konklusion spricht dagegen, (9) im Sinne einer Anzahlaussage auszulegen; gleichwohl gilt die Leugnung der Vielheit als genuin Parmenideische Lehre. Und daran ist wohl festzuhalten, trotz des Verdikts von Mourelatos (in The Route of Parmenides, S. 130) gegen die platonisch-aristotelische Version der Philosophie Parmenides': ,, it would be wrong and arbitrary to interpret Parmenides' ,Truth ' as an holistic monism." Wir werden es heute kaum besser wissen können als Platon und Aristoteles, die den gesamten Text vorliegen hatten und darüber, daß Parmenides eben dies u.a. meinte, nicht im Zweifel waren. (In

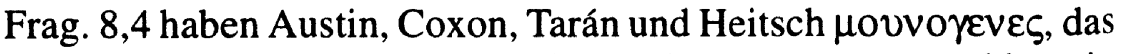
man durch ,einzig“ übersetzen kann; das Wort stützt unabhängig von $\varepsilon v$ in 8,6, und wesentlich besser als dieses, die Aufnahme von 
(9) als Anzahlaussage unter die Parmenideischen Kernthesen. Diels/Kranz und Gallop haben aber stattdessen ov $\lambda$ o $\mu \varepsilon \lambda \varepsilon \varsigma$ in Frag. 8,4.)

Außerdem: Mag die Wahrnehmung, die uns das Vorhandensein von Vielheit und Veränderung in ihren Inhalten suggeriert, bezüglich dieser Inhalte illusionär sein; es ist doch nicht zu leugnen, daß es viele und sich als Wahrnehmungen (z.B. in ihrer Intensität) verändernde Wahrnehmungen gibt. Sind diese nicht seiend, so gibt es Nichtseiendes; sind sie dagegen seiend, so ist nicht mehr zu bestreiten, daß Vielheit und Veränderung im Seienden vorhanden sind. Wie kann Parmenides zwischen den Hörnern dieses Dilemmas hindurchschlüpfen?

Es gibt einen Weg. Nehmen wir an, Parmenides intendiert von vornherein nur von objektiven Entitäten zu sprechen, wenn er seine ontologischen Behauptungen aufstellt, d.h. nur von Entitäten, die ihrer Existenz und Beschaffenheit nach unabhängig sind von der Existenz und Beschaffenheit erkennender Subjekte und deren Erkenntnisakte. Wenn er also sagt, daß es Nichtseiendes nicht gibt, so meint er, daß es objektives Nichtseiendes nicht gibt, d.h. daß alles Objektive seiend (benennbar oder erkennbar, bzw. aktual) ist. Erkenntnissubjekte (wie Parmenides und wir alle) und deren Erkenntnisakte (einschließlich Wahrnehmungen) sind keine objektiven Entitäten; deren Vielheit und Veränderung blieben also unangetastet, wenn Parmenides daraus, daß es unter den objektiven Entitäten nur aktuale gibt, folgerte, daß es keine objektive Veränderung und keine objektive Vielheit gibt.

Die oben angegebenen Deduktionen (a) und (b) bleiben korrekt, wenn man das Wort „Objekt" in ihnen im Sinne von ,,objektive Entität" versteht, statt ,Hinsicht“ und „,Sachverhalt" ,,objektive Hinsicht" und ,objektiver Sachverhalt" setzt, etc. Man folgert dann daraus, daß alle objektiven Entitäten aktual sind und es aktuale objektive Entitäten gibt, daß es keine objektive Veränderung und keine objektive Vielheit gibt. Z.B.:

$\left(a^{\prime}\right)$ Angenommen die objektive Entität $x$ ist bzgl. der Zeitpunkte $t$ und $t^{\prime}$ in der objektiven Hinsicht $f$ verändert ... In beiden Fällen besteht also ein gewisser objektiver Sachverhalt nicht; also gibt es einen nichtbestehenden objektiven Sachverhalt; also gibt es Objektives, das nicht real ist. Es ist jedoch laut Annahme alles Objektive 
real. Folglich gibt es eben keine objektive Entität $x$, keine Zeitpunkte $t$ und $t^{\prime}$ und keine objektive Hinsicht $f$, so daß gilt: $x$ ist bzgl. $t$ und $t^{\prime}$ in $f$ verändert. Also gibt es keine objektive Veränderung.

Parmenides zu unterstellen, daß er seine ontologischen Thesen nur auf den Bereich des Objektiven bezieht, ist nicht so problematisch, wie es auf den ersten Blick scheinen mag. Nicht nur entgeht er damit manifesten Absurditäten, sondern die Unterscheidung subjektiv-objektiv kannte bereits sein Lehrer Xenophanes; sie ist in dem berühmten erkenntniskritischen Fragment 34 eindeutig impliziert. Aufgrund der Unterstellung ergibt sich, da $\beta$ Parmenides uns (die vielen anderen aktualen sich wandelnden Erkenntnissubjekte!) nicht etwa auffordert zu akzeptieren, daß es genau ein unveränderlich Seiendes (Aktuales) gebe und sonst nichts, sondern nur, daß genau ein objektives unveränderlich Seiendes und sonst nichts $\mathrm{Ob}$ jektives da sei. Aus Parmenides' (entsprechend gedeuteter) Prämisse ist diese Konklusion tatsächlich logisch ableitbar. Gleichwohl sie weniger absurd ist als die andere, wird man nicht bereit sein, sie zu akzeptieren, sondern vielmehr wiederum die Falschheit der Prämisse konstatieren, auf der sie beruht. Es gibt eben doch objektive nichtaktuale Entitäten. Parmenides freilich war dieser Ausweg versperrt. Es stand ihm nicht frei, die Prämisse zu leugnen; er hielt sie ja für evident. Daß sie nur in bestimmten, für die Folgerbarkeit der Konklusion unzureichenden Deutungen (z.B. D2(5) + Def1) evident ist, entging ihm wegen seiner undifferenzierten monolithischen Seinskonzeption. Die notwendige analytische Arbeit der Differenzierung der Seinsbegriffe, zu der Parmenides den Anstoß gab, haben Platon und vor allem Aristoteles vorangetrieben. Man kann aber selbst zum jetzigen Zeitpunkt nicht sagen, daß diese Arbeit abgeschlossen wäre.

VII. Abschließend möchte ich auf eine neuere einflußreiche Parmenides-Interpretation kritisch eingehen. Ich meine diejenige von Ch. H. Kahn in ,,The Thesis of Parmenides" (The Review of Metaphysics 22 (1969), S. 700-724). Nach Kahn ist die These, von der Parmenides bei der Begründung seiner skandalösen Aussagen bzgl. Veränderung und Vielheit ausgeht: ,,that something must be the case in the world for there to be any knowledge or any truth" (S. 714). 
Dies soll der Gehalt von Frag. 2,3 sein (ich würde diese Zeile als alternative, elliptische Formulierung von D2(5) ansehen), den er aber an anderer Stelle (S. 711) anders angibt: ,Parmenides is making the obvious, but not entirely trivial claim that whatever we know, whatever can be known, is - and must be - determinately so, that it must be actually the case in reality or in the world." Kahn zeigt überzeugend, daß man Frag. 2,3 so lesen kann; die beste Lesart ist die seinige aber wohl nicht. Denn auf ihrer Basis kann man nicht wirklich befriedigend erklären, wie Parmenides aufgrund seiner Prämisse zu seiner Leugnung aller Veränderung und Vielheit kommt. Aus einer analytisch wahren Prämisse folgt nur analytisch Wahres, was Parmenides' Konklusion gewiß nicht ist; Parmenides' Prämisse ist aber in Kahns Deutung analytisch wahr und kann anders als bei der Deutung, von der wir hier schließlich ausgegangen sind - nicht auch in einem metaphysisch ,,gefährlichen“ zweiten Sinn als synthetische Aussage gelesen werden. Kahn muß also unterstellen, daß Parmenides stillschweigend weitere synthetische (und problematische) Prämissen unterschiebt, bzw. daß er grob falsch schließt, um zu erklären, wie Parmenides zu seiner Konklusion kommt. Demgegenüber wird bei unserer Deutung Parmenides' Fehler minimiert; er hat korrekt geschlossen und keine heimlichen Prämissen gebraucht, er hat nur den zunächst gebrauchten Sinn eines Wortes in seiner explizit angegebenen Prämisse mit einem anderen Sinn dieses Wortes verwechselt, was, wie dargelegt, nicht ohne eine gewisse Zwangsläufigkeit geschah.

Kahn geht nicht auf die Stelle ein, auf die wir uns hier hauptsächlich bezogen haben: Frag. 8,36-41 (der folgernde Übergang von (5) auf (6)). Dort aber nennt Parmenides noch einmal seine Prämisse in Zeile 36/37, nämlich (5), und es ist schwer zu sehen, wie dieses dasselbe besagen kann - wovon aber auszugehen ist - wie Frag. 2,3, wenn man letzteres in Kahns Deutung nimmt. Frag. 6,1, Frag. 7,1 und Frag. 8,46 erscheinen zudem in Kahns einheitlich veridikaler Interpretation (S. 711) von $\varepsilon \sigma \tau \iota, \varepsilon \imath v \alpha l$ und $\varepsilon 0 v$ als platteste Tautologien, die auch nicht einen Teil dessen besagen, was nach Kahn in Frag. 2,3 steht. Wie ist das Auftreten dieser Platitüden zu erklären, was ist ihre Funktion? Dieses Problem bleibt bei Kahn offen.

Schließlich ein Wort zum veridikalen Sinn von Elval (ohne 
prädikative Ergänzung), auf die Kahn seine Parmenides-Interpretation aufbaut. Außer Frage steht, daß die Verwendung von $\varepsilon ı v \alpha \iota$ in diesem Sinn im Altgriechischen gang und gäbe ist, und das ist im Blick auf die Frage, ob denn die Schlußfolgerungen (a) und (b) in V. Parmenides auch nur ungefähr zugeschrieben werden können, eine sehr willkommene Tatsache. Denn im veridikalen Sinn genommen besagt $\varepsilon \imath v \alpha \iota$ soviel wie ,,der Fall sein“, und die Objekte, von denen es wahrheitsgemäß ausgesagt werden kann, sind: bestehende (aktuale) Sachverhalte, d.h. Tatsachen.

Es zeigt sich also, daß der veridikale Sinn von elval nichts anderes ist als eine Spezialisierung des existentiellen Sinns von Elval, nicht etwa, wie Kahn offenbar meint (S. 711), ein anderer Sinn neben dem existentiellen Sinn; denn ,, $\mathrm{x}$ ist der Fall“ besagt ja nichts anderes als , $x$ ist ein aktualer (d.h. in einem speziellen Sinn existierender/seiender) Sachverhalt"; $\varepsilon$ lv $\alpha$ l im veridikalen Sinn ist schlicht $\varepsilon t v \alpha \mathrm{l}$ im Sinne von Aktualsein bezogen auf Sachverhalte. Ohne es zu ahnen, gibt dies Kahn auf der ersten Seite seines Aufsatzes gewissermaßen zu: ,For he [Parmenides] is the first to have articulated the concept of Being or Reality [!] as a distinct topic for philosophical discussion." Nun läßt sich eine einheitlich veridikale Deutung aller Vorkommnisse von عiv $\alpha \mathrm{l}$ und seiner grammatikalischen Abwandlungen in Parmenides' Lehrgedicht, wie Kahn sie vertritt, wohl kaum aufrechterhalten (einen Grund dafür habe ich am Ende des letzten Absatzes genannt); wo sie aber am Platze ist - was aber gerade nicht dort ist, wo $\varepsilon \sigma \tau l$ ohne prädikative Ergänzung auftritt: da vertritt es vielmehr den Existenzquantor -, dort wird sie problemlos durch unsere Deutung von $\varepsilon l v \alpha l$ im Sinne von Def2 mitumfaßt. 


\section{LITERATUR}

Austin, S.: Parmenides. Being, Bound, and Logic, Yale University Press: New Haven/London 1986.

Coxon, A. H.: The Fragments of Parmenides, Van Gorcum: Assen/ Maastricht 1986.

Gallop, D.: Parmenides of Elea, University of Toronto Press: Toronto/London 1984.

Guthrie, W. K.: A History of Greek Philosophy, Bd. VI, Cambridge University Press: Cambridge 1981.

Heitsch, E.: Parmenides: Die Fragmente, Artemis: München/Zürich 1991.

Kahn, Ch. H.: ,,The Thesis of Parmenides“, The Review of Metaphysics 22 (1969), S. 700-724.

Mourelatos, A. P. D.: The Route of Parmenides, Yale University Press: New Haven/London 1970.

Tarán, L.: Parmenides, Princeton University Press: Princeton, N. J., 1965. Tegtmeier, E.: Grundzüge einer kategorialen Ontologie, Alber: Freiburg/München 1992. 